\title{
A Duplicated Forgery Detection Fusion Algorithm using SIFT and Radial-Harmonic Fourier Moments
}

\author{
Yanfen Gan ${ }^{\mathrm{a}}$, Jimlee Chung ${ }^{\mathrm{b}, *}$, Janson Young ${ }^{\mathrm{c}}$, Zixin $\mathrm{Hu}^{\mathrm{b}}$, Jinhong Zhao ${ }^{\mathrm{b}}$ \\ ${ }^{a}$ School of Computer Science and Technology, Guangdong University of Foreign Studies South China Business College, Guangzhou, 510545, China \\ ${ }^{b}$ School of information engineering, Guangdong Mechanical \& Electrical College, Guangzhou, 510550, China \\ ${ }^{c}$ School of Computers, Guangdong University of Technology, Guangzhou, 510006, China
}

\begin{abstract}
This paper proposed an algorithm that fused block-based algorithm (Radial Harmonic Fourier Moments, RHFMs) and SIFT algorithm (Scale Invariant Feature Transform, SIFT) for the duplicated forgery detection, also called copy-move forgery detection. It can effectively detect forgery image. Firstly, the SIFT algorithm is proposed to extract feature points from the pre-processing image. Subsequently, the nearest-neighbor ( $2 \mathrm{NN}$ ) test, adaptive Euclidean distance and Random sample consensus (RANSAC) are applied to remove most of the mismatched feature points and get the candidate inlier matches. The affine matrixes based on the RANSAC are obtained by the candidate inlier matches. Then, Radial Harmonic Fourier Moments is proposed to extract invariances of the candidate inlier matches in circle blocks. The propagated criterion is calculated by affine matrix and circular feature of the inlier matches. The Simple Linear Iterative Clustering (SLIC) is presented to segment the host image into texture patches. Like pixel propagating, the circular block is slid in the corresponding texture blocks that the texture blocks contain the corresponding inlier matches to get more corresponding matches. Finally, some geometric image operations, such as dilation, are employed to eliminate the small holes or isolated pixels. A series of experiments showed that the proposed fusion algorithm can achieve superior performances than those of the moment invariant algorithms under various geometric transformations.
\end{abstract}

Keywords: duplicated forgery detection; scale invariant feature transform; radial harmonic fourier moments

(Submitted on October 17, 2017; Revised on November 23, 2017; Accepted on December 29, 2017)

(C) 2018 Totem Publisher, Inc. All rights reserved.

\section{Introduction}

Nowadays, a forgery image can be easily created to represent the realistic image without any professional skills. If these forgery images are used in news reports, commercial contracts, law evidences, or academic researches, it will cause serious social problems. In order to solve this problem, digital image forensics has emerged to discern true or false images. There are several types of forgeries. One of the most common type is duplicated forgery or so-called copy-move forgery [23]. In this type, a part of the image is copied and moved to another part of the image that aims to cover some object by one's intention. And also, the forger will apply blur degradation, geometric transforms or arbitrary contrast changes on the duplicate regions to achieve his aim.

The simplest duplicated forgery detection algorithm to detect whether an image has been forged is exhaustive search algorithm. But, the efficiency of exhaustive search is too low to use in forgery detection. Fridrich et al. [8] proposed an algorithm that divides the image by overlapping and square blocks. The discrete cosine transform (DCT) coefficients of those blocks are computed to identify the original region and its duplicate region. Similarly, PCA (Principal Component Analysis) [13], and SVD (Singular Value Decomposition) [11] are image features that are also employed to represent the blocks. Luo et al. [12] proposed the main shift vector to identify the possible duplicate region. It can resist various forms of post region duplicate image processing. For keypoint-based algorithms, Amerini et al. [3] proposed a novel SIFT based on J-Linkage, which can achieve accurate forgery localization. Ardizzone et al. [4] presented a hybrid algorithm to fuse keypoint-based algorithms with Delaunay triangulation. For block-based algorithms, Tu et al. [10] improved Zernike moments by combining

\footnotetext{
* Corresponding author.

E-mail address: junliuzhong@foxmail.com
} 
it with DWT to boost the feasibility and efficiency. The main defect of block-based algorithms is the lack of the resisting of the scaling transforms. Wo et al. [18] and Emam et al. [7] proposed the polar complex exponential transform (PCET) with multi-radius block to extract the feature of each point. But, its efficiency is low. One of the most famous solution are the keypoint-based algorithms, such as scale invariant feature transform (SIFT) [2,9,20] and Speeded Up Robust Features (SURF) [16]. The keypoint-based algorithm has high detection accuracy, but needs the auxiliary matting algorithm to fill the detected regions.

To address the above defect of algorithms, we propose a novel algorithm that fuses the merits of the keypoint-based algorithm and block-based algorithm to achieve a higher detection accuracy and efficiency. In [21], Zheng et al. also proposed fusion of block and keypoint based algorithms. However, the algorithm of [21] relies on the number of keypoints of segmentation to identify whether the region is the suspicious region, which is unreliable. In our proposed algorithm, the keypoint-based algorithm, namely SIFT, extracts the feature point and preliminarily locates the coarse regions. Then, the Radial Harmonic Fourier moment (RHFMs) extracts the features of the patches that contain the SIFT points to obtain the threshold of the candidate matches. Finally, the block is employed to traverse the above patches with the matching threshold to indicate the forged regions.

The rest of paper is organized as follow: the frame of detection algorithm, image feature matching, and preliminary filtering algorithm are proposed in section 2 and section 3, respectively. Further filtering by using RHFMs is presented in section 4. The analysis of the experiment results is given in section 5. Finally, the conclusion is given in section 6.

\section{The Frame of Detection Algorithm}

There are a large number of the algorithms proposed for duplicated forgery detection. These algorithms are often divided into two forms , either block-based algorithms or keypoint-based algorithms [6]. Most of these algorithms follow similar processing steps. The feature extraction is the main difference between the two kind algorithms. The fusion algorithm is proposed to merge the merits to better filter out the outliers. The steps of the algorithm are given as follow.

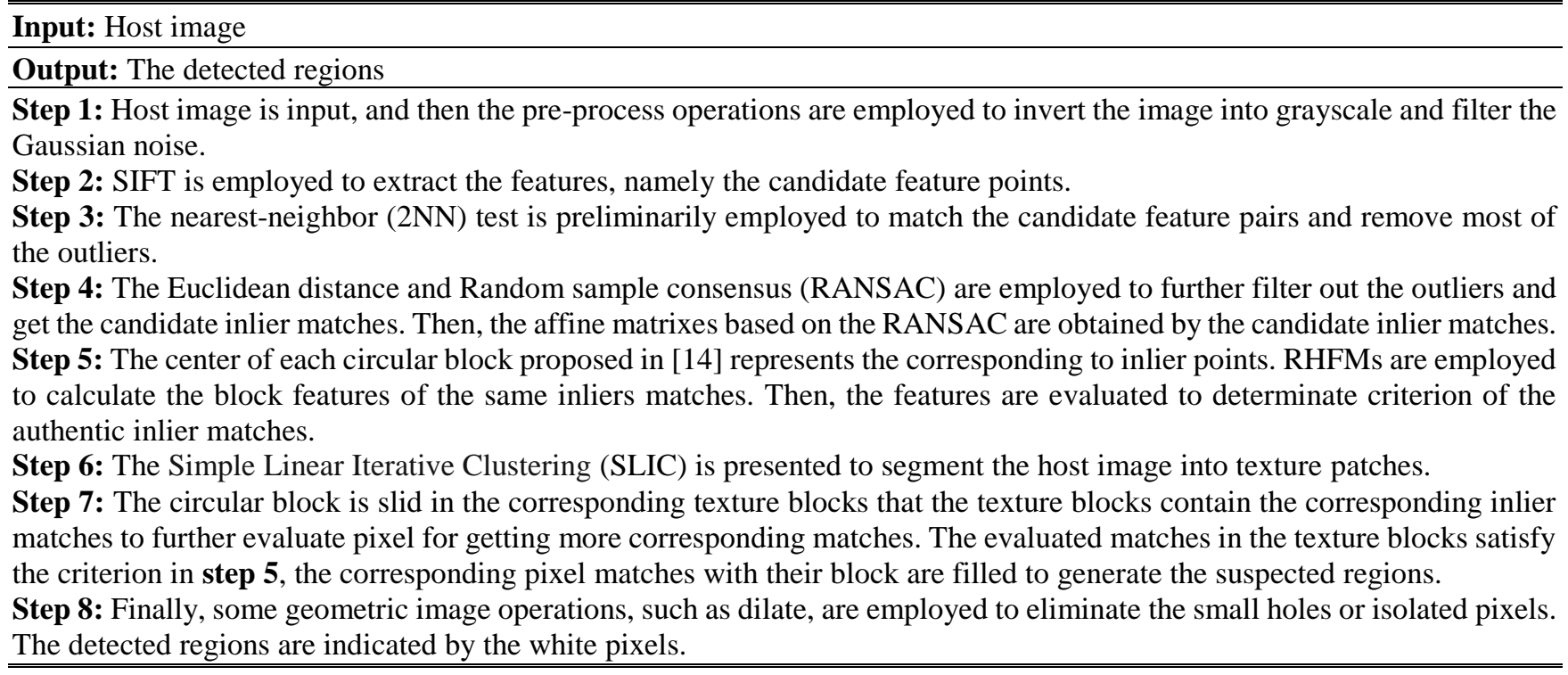

Steps 3-5 and 7 of our proposed algorithm are given with a detailed description as follow. Other steps are general operations in various algorithms [2,14,22].

\section{The image feature matching and filtering (step 3 - 4)}

Figure 1 shows the process of the matching and filtering. Figure 1(a) shows the original image. Figure 1(b) shows the copymove forged image under translation distortion. After pre-processing operations, SIFT is employed to extract the local maxima and minima pixels as the image features. It is also expressed as the candidate feature point. Then, the feature point matching and filtering algorithm [22] are proposed to obtain the inlier matches and remove the outlier matches. Some effective matching algorithms are proposed for duplicated forgery detection [6]. Christlein et al. [5] applied the Kd-tree to classify the maxima and obtain the candidate matches. But, the efficiency of Kd-tree is low. The 2NN test [2] is an alternative algorithm to make 
the matching simpler. It is employed to evaluate the correlation or the distance between each candidate feature point and the rest of the feature points, and then are sorted by Lexicographic sorting. The ratio threshold determinates the point matches. Subsequently, the candidate inliers (matches) are preliminarily filtered by the Euclidean distance. Figure 1(c) shows the candidate feature point matching by employing $2 \mathrm{NN}$ test and filtering algorithm with Euclidean distance. It is observed that there are some mismatched feature-point pairs in Figure 1(c). Finally, RANSAC [17] is a good tool for classifying the clusters as shown in Figure 1(d). It is observed from Figure 1(d) that the feature-point pairs (inlier matches) are truly classified into 3 clusters.

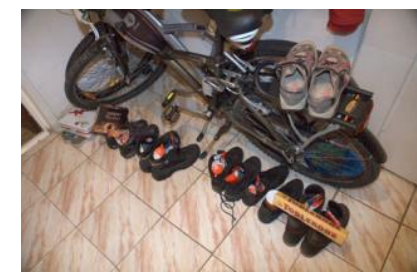

(a)

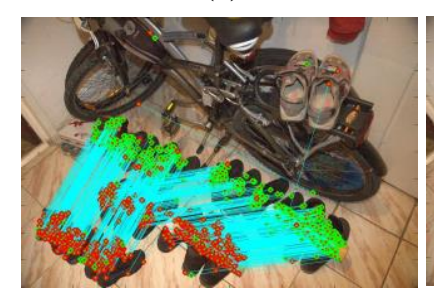

(c)

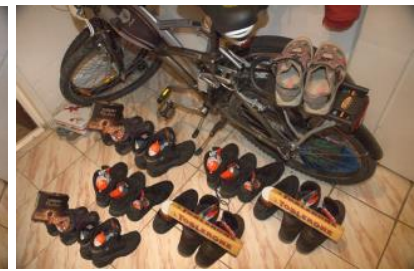

(b)

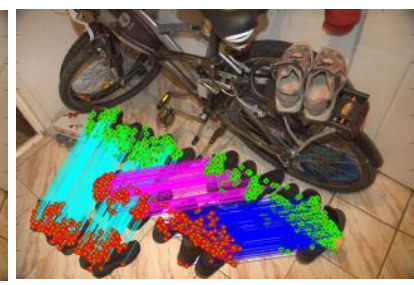

(d)

Figure 1. The extracting and matching of the candidate feature points. (a) original image; (b) copy-move image; (c) matching and filtering with $2 \mathrm{NN}$ test and Euclidean distance; (d) classification with RANSAC.

The $2 \mathrm{NN}$ test is defined as (1).

$$
d l_{2} / d l_{1}<T_{1} \text {, where } T_{1} \in(0,1)
$$

where $d l_{1}$ and $d l_{2}$ represent the closest distance and the $2^{\text {nd }}$ closest distance of the candidate feature matches, $T_{1}$ is a threshold value of the distance ratio. Based on the large amount of experimental analysis, $T_{1}$ is set to 0.5 to get more matches. After the $2 \mathrm{NN}$ test, there are still some candidate matches that are close to each other. These nearest-neighbor matches are regarded as the mismatches or outliers. Pun et al. [14] proposed the 'Haar' wavelet to calculate the frequency distribution of the image. The adaptively Euclidean distance with $P_{l f}$ is proposed for candidate matches filtering. The adaptive Euclidean distance is defined as (2).

$$
\operatorname{dist}(U, V)= \begin{cases}P_{l f} \times \sqrt{0.01 \times M \times N}, & P_{l f}>0.5 \\ P_{l f} \times \sqrt{0.02 \times M \times N}, & P_{l f} \leq 0.5\end{cases}
$$

where $U$ and $V$ are the candidate matched feature points belonging to two clusters, $M$ and $N$ are the size of the image, $P_{l f}$ is the coefficient of frequency distribution [14]. As shown in Figure 1(c), most of the outliers that contain nearest-neighbor keypoint matches are removed. The candidate matches are reserved to locate the forged region. Most of the candidate matches concentrate on the copy move regions. There are also some candidate matches that are false matches in Figure 1(c). RANSAC [17] is a good tool for auto-classification. We applied it to further classify the candidate keypoint matches into inlier matches and remove the above false matches.

\section{The further filtering by Using Radial Harmonic Fourier Moments (step 5)}

Based on steps 1-4, we can obtain the candidate keypoint matches (inlier matches). We also obtain the affine matrix between two matching clusters by using RANSAC. Then, we employ the circular block to extract the local feature of inlier points. The center of each circular block proposed in [22] represents the corresponding inlier point. Qin et al. [15] proposed Radial Harmonic Fourier moment (RHFMs), to construct the deformation model that has the rotation invariant and orthogonal features. The algorithm can be applied to the duplicated forgery detection and obtain a good robustness and high detection accuracy under rotation operations. Hence, we proposed RHFMs to calculate the block features of the inliers matches. Finally, the features are evaluated to determinate threshold of the authentic inlier matches. 


\subsection{The review of the Radial Harmonic Fourier Moments}

RHFMs are consisted of the radial kernel and complex exponential function [14]. The kernel of RHFMs consists of the three modes, which are real number, triangular sine, or Triangular cosine. The kernel is so simple to compare other state-of-the-art moments. The kernel of RHFMs is orthogonal moment. It has many superior performances, such as good rotation invariant, certain degree scaling invariant, simple computational complexity and less data redundancy. It is a good tool for duplicated forgery detection. The RHFMs function is defined as (3).

$$
\Phi_{n l}=\int_{0}^{2 \pi} \int_{0}^{1} f(r, \theta) K_{n}(r) \exp (-i l \beta) r d r d \beta
$$

where $K_{n}(r)$ is the kernel of the RHFMs, $n$ is the order and $l$ is the repetition of RHFMs, $k=0,1,2, \ldots, \infty, l=1,2, \ldots, \infty, i^{2}=-$ $1, r$ is length defined in the unit circle and $\beta$ is the projection angle. The radius $r$ and projection angle $\beta$ are defined as follows.

$$
\begin{gathered}
r=\left\|x_{i}-x_{0}, y_{i}-y_{0}\right\|_{2} \\
\beta=\arctan \left(\frac{y_{i}}{x_{i}}\right)
\end{gathered}
$$

where $x_{i}, y_{i}$ are the coordinates of the pixel in the sliding circle block that is defined in [22], $x_{0}, y_{0}$ is the origin coordinate of the circle block, which is defined in the center of the block. The kernel function $K_{n}(r)$ is defined as (6).

$$
K_{n}(r)= \begin{cases}\frac{1}{\sqrt{r}} \quad, \text { where } n \text { is equal to } 0 \\ \left.\sqrt{\frac{2}{r}} \sin [(n+1) \pi r)\right], \text { where } n \text { is odd number } \\ \sqrt{\frac{2}{r}} \cos (n \pi r) \quad, \text { where } n \text { is even number }\end{cases}
$$

It is easy to prove that the kernel of the RHFMs has the orthogonality. The radius of the circular block is set to a larger value to reduce computation errors. In our proposal, the radius of the block is set to 10 .

\subsection{The definition of the RHFMs with even order}

To summarize, RHFMs have three forms, which consists of 0 order, odd order and even order as given in Formula (6). Polar cosine transform has the relevant better performance than that of the polar sine transform [19]. In our proposed algorithm, the even order of the RHFMs with cosine is proposed to construct the geometric invariant moments. It supposed that $\beta$ is the projection angle to the origin coordinate in the circle block. $R$ is the radius of the circular block. $\theta$ is the rotation angle of the forged region corresponding to the copied region. The rotation angle of the copy move region is $\phi=\beta+\theta$, and the derivate of $\phi$ is $d \phi=d \beta$. It is also supposed that the original projection function $f(r, \beta)$ is clockwise rotated $\theta$ angle to $\phi$. The rotation invariant of RHFMs $\Phi_{n, l}^{r}$ is defined as (7).

$$
\begin{aligned}
& \Phi_{n, l}^{r}=\int_{0}^{2 \pi} \int_{0}^{R} f_{r}(r, \phi) K_{n}(r) \exp (-i l \phi) r d r d \phi \\
& =\int_{0}^{2 \pi} \int_{0}^{R} f(r, \phi-\theta) \sqrt{\frac{2}{r}} \cos (n \pi r) \exp (-i l(\beta+\theta)) r d r d \beta \\
& =\mathrm{e}^{(-i l \theta)} \int_{0}^{2 \pi} \int_{0}^{R} f(r, \beta) \sqrt{\frac{2}{r}} \cos (n \pi r) \exp (-i l \beta) r d r d \beta \\
& =e^{-i l \theta} \Phi_{n, l}
\end{aligned}
$$


where $\Phi_{n, l}^{r}$ is the product of the original moment $\Phi_{n, l}$ with angular component $e^{-i l \theta}$, the rotation invariant moment is defined in (8).

$$
\left|\Phi_{n, l}^{r}\right|=\left|e^{-i l \theta} \Phi_{n, l}\right|=\left|\Phi_{n, l}\right|
$$

where $\mid$ means the amplitude of the RHFMs. It is easily observed that the amplitude of the RHFMs has the rotation invariance. It is easy to construct the rotation invariant in a circular block.

\subsection{The local feature extraction of inlier points with the adaptive circular block}

From the above steps, we obtain the affine matrix between two matching clusters. From Eq. (8), we can obtain the rotation invariant of the block. We fuse Eq. (8) and the scaling factor that is obtained in affine matrix to adaptively adjust the radius of the circular block. The adaptive adjustment of the radius is defined as follow.

$$
C_{r}=\text { Floor }\left[10 \times \lambda_{s}\right]
$$

where Floor[] denotes the floor integer operation, 10 denotes the base radius of circular block, and $\lambda_{s}$ denotes the scaling factor between two clusters. One cluster is randomly chosen, such as cluster 1, as the base cluster. The block radii of other inlier clusters are adaptively adjusted by using Eq. (9). The Eq. (8) can be rewritten as (10) to calculate the features of the circular block.

$$
\Phi_{n, l, s}=\left|\sum_{r \leq 10 \times \lambda s} \Phi_{n, l, r}\right|
$$

where $\Phi_{n, l, s}$ denotes the feature of the corresponding the inlier, $\Phi_{n, l, s}$ denotes the RHFMs feature of the corresponding pixel in circular block. When the circular block is applied to calculating the base cluster, $\lambda_{s}=1$ and the base radius is kept as 10 .

\subsection{The base threshold of the authentic inlier matches}

Based on the Eq. (8) - (10) and affine matrix, we can calculate the block feature of the inlier matches of the all clusters to obtain the correlation coefficient of the candidate matches. We define the block feature of the all clusters as $C_{f}=\left\{C_{f, 1}, C_{f, 2}, \ldots, C_{f, i}, \ldots, C_{f, m}\right\} . C_{f, i}$ denotes the $n o . i$ cluster, $i=1,2, \ldots, m$, and $m$ denotes the number of the cluster. The no.i cluster can be defined as $C_{f, i}=\left\{C f_{i, 1}, C f_{i, 2}, \ldots, C f_{i, n}\right\} . C f_{i, j}$ denotes the block feature of the no.j inlier point, and $n$ denotes the number of inlier in no. $i$ cluster. It is important to note that the inlier matches may be one to one, one to many, or many to many forms. Figure 2 shows the illustrated forms of cluster matches. In Figure 2(a), we can observe that it is one to one cluster matches. We can randomly select one cluster as the base cluster, such as cluster 1 . We define correlation coefficient of the corresponding inlier matches in two clusters (cluster 1 and 2) by using the first derivative. The mean value of the first derivative vector is defined as follow.

$$
\overline{\nabla\left(C_{f, 1,1^{\prime}}\right)}=\operatorname{mean}\left(\nabla\left(C_{f, 1^{\prime}} / C_{f, 1}\right)\right)=\operatorname{mean}\left(\nabla\left(\frac{C f_{1^{\prime}, 1}}{C f_{1,1}}, \ldots, \frac{C f_{1^{\prime}, n}}{C f_{1, n}}\right)\right)
$$

where $\nabla$ denotes the first derivative, $\overline{\nabla\left(C_{f, 1,2}\right)}$ denotes the mean value of the cluster vector derivative. Other forms, such as one to many, are similar to Eq. (11). The generalized equation that contains one to one, one to many and many to many forms is defined as follow.

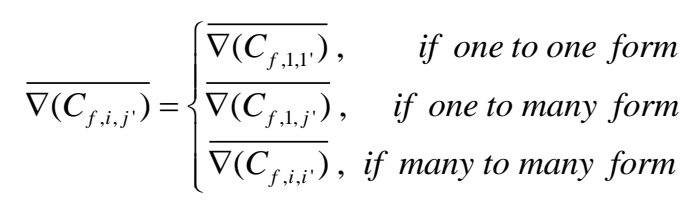


where $\overline{\nabla\left(C_{f, 1, i}\right)}$ denotes the mean value of the cluster vector derivative. Eq. (12) can be the evaluated threshold to determinate the authentic inlier matches.

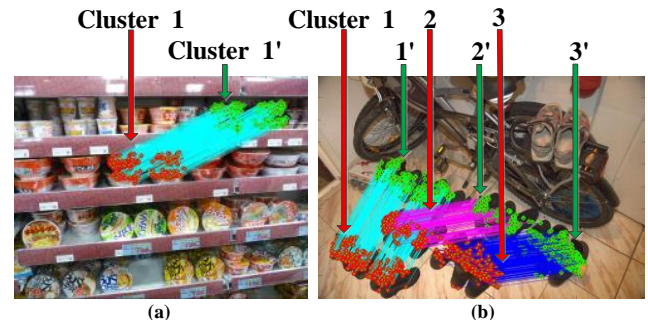

Figure 2. The illustrated forms of cluster matches: (a) one to one cluster; (b) many to many clusters.

\section{The matting operation to generate the suspected regions (step 6 and 7)}

From above steps, we can obtain the inlier matches. However, as shown in Figure 2, the number of the matches is not enough to fill the suspicious regions. The large number of new matches needs to generate in order to implement the matting. Based on the above analysis, the block feature of candidate matches satisfies the criterion, as defined in (13).

$$
\left\{\begin{array}{l}
1 \geq M t_{i, p, q} \geq \overline{\nabla\left(C_{f, i, i^{\prime}}\right)}, \text { if } \overline{\nabla\left(C_{f, i, i^{\prime}}\right)} \leq 1 \\
\overline{\nabla\left(C_{f, i, i^{\prime}}\right)} \geq M t_{i, p, q} \geq 1, \text { if } \overline{\nabla\left(C_{f, i, i^{\prime}}\right)}>1
\end{array}\right.
$$

where $M t_{i, p, q}$ denotes the no.p and no.q pixel in the detected image. Based on Eq. (13), we can search the candidate pixel matches on the whole image. However, global search has low efficiency and low precision, especially for many to many forms. We cannot identify which cluster the pixels are. So, we employed the Simple Linear Iterative Clustering (SLIC) [1] to segment the image into meaningful patches as shown in Figure 3. Each patch is based on the texture segmentation. Then, we map the inlier matches into segmented patches. From Figure 4(a), it is observed that the patches that contain the inlier matches cover the all ground-truth regions. We only search these patches to fill the suspicious pixels or regions. So, the circular block is employed to search all pixels in the patch that belongs to cluster $i$. Then, the affine matrix is employed to calculate the block feature of the corresponding approximate pixel that belongs to the corresponding cluster $i$ '. If the block features of compared pixels satisfy the criterion that is defined in Eq. (13), the compared pixels are as matches. The pixel matches are filled in white colour to indicate the detected regions. It is a propagated stage as shown in Figure 4-(b).

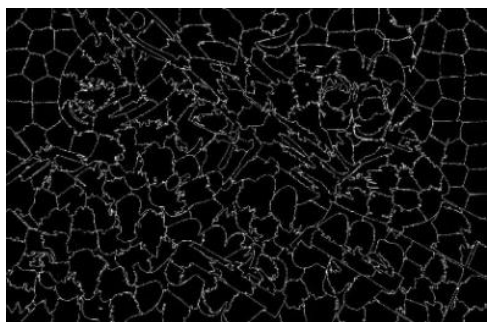

Figure 3. Image SLIC segmentation

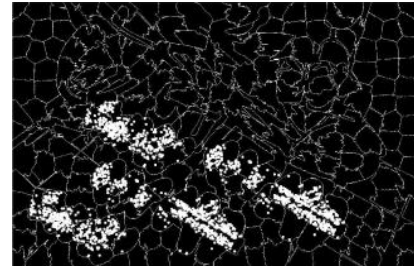

(a)

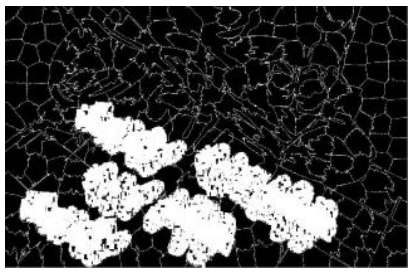

(b)

Figure 4. The matting result of propagated pixel: (a) the inlier matches located in SLIC patches; (b) the propagated pixel for region matting.

\section{The analysis of the experimental results}

In this section, extensive experiments are conducted to validate that the proposed copy-move forgery detection algorithm performs better robustness than the state-of-the-art algorithms. In the following experiments, the benchmark databases [6] of duplicated forgery detection were applied to the experiment analysis. 


\subsection{The geometric performances of our proposed algorithm}

To comprehensively evaluate the strong robustness of the proposed algorithm, a large number of the copy move images are selected from the dataset. The tested images [6] contain the artifacts, plants, animals and landscapes, etc. The tested images implemented the geometric distortions, such as translation (plain), rotation, small-scale scaling, down-sampling operations etc. Figure 5 shows the experimental results. Figure 5(a1)-(a4) show the copy-move images. Figure 5-(a1) shows the image implemented with the translation transform. Figure 5-(a2) shows the red tower image implemented with a rotation angle of $60^{\circ}$. Figure 5-(a3) shows the host image implemented with the scale factor of 1.09. Figure 5-(a4) shows the host image implemented with the down-sampling factor of 0.5. Figure 5(b1)-(b4) show the detected results of the proposed algorithm that are depicted with white color. Figure 5(c1)-(c4) show the ground-truth regions corresponding to the Figure 5(a1)-(a4).

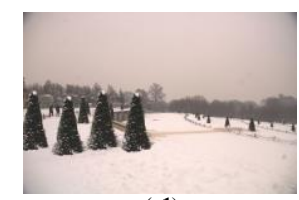

(a1)

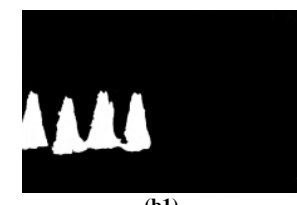

(b1)

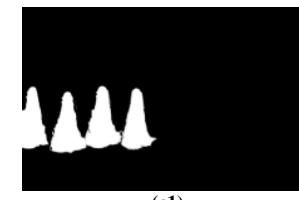

(c1)

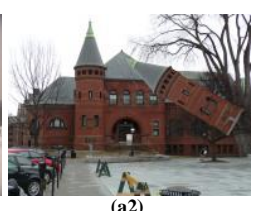
(a2)

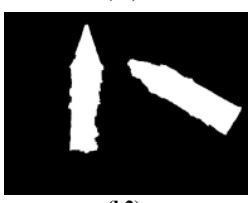

(b2)

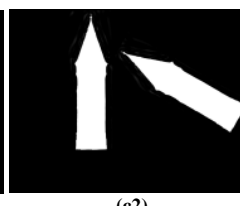

(c2)

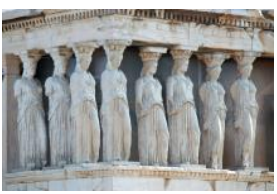

(a3)

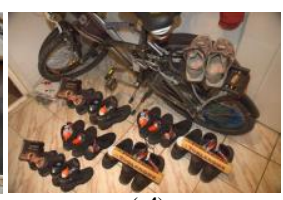

(a4)

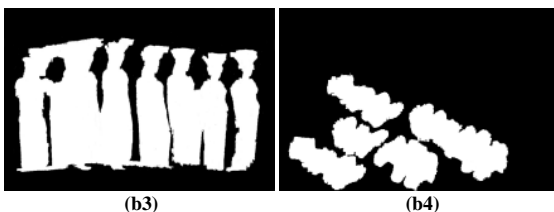

(b3)

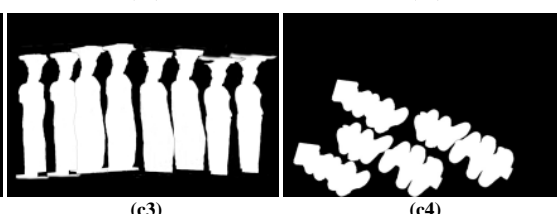

(c3)

(c4)

Figure 5. The experimental results of the proposed algorithm: (a) the copy-move images; (b) the experimental results of the proposed algorithm; (c) the ground-truth regions.

Comparisons between Figure 5(b1)-(b4) and Figure 5(c1)-(c4) show that the similarity between the proposed algorithm and the ground-truth regions are over $90 \%$. The proposed algorithm has superior performances for duplicated forgery detection.

\subsection{The performance comparisons between the proposed algorithm and the state-of-the-art algorithms}

To evaluate the performance of the proposed algorithm and the state-of-the-art algorithms, the precision and recall [22] as two criteria evaluated criterion. $F_{1}$ score combines both precision and recall as defined in (14).

$$
F_{1}=2 \times \frac{\text { precision } \times \text { recall }}{\text { precision }+ \text { recall }}
$$

The $F_{1}$ score can reflect the comprehensive performance of the algorithm. When the precision, recall and $F_{1}$ score get close to 1 , the better they perform. To quantitatively evaluate the performances of the proposed algorithm and the state-of-theart algorithms, the experimental images were tempered by plain, rotation, scaled transforms and down-sampling operations at the pixel level. The number of each type of operation is listed in Table 1.

Table 1. The type and the number of experimental images

\begin{tabular}{|c|c|c|c|c|}
\hline The forgery type & plain & rotation degree & scaling factor & Down-sampling \\
\hline Experimental image & Translation & $\begin{array}{c}2^{\circ}, 4^{\circ}, 6^{\circ}, 8^{\circ}, 10^{\circ}, 20^{\circ}, \\
60^{\circ} \text { and } 180^{\circ} .\end{array}$ & $\begin{array}{c}0.8,0.91,0.95,0.99,1.01 \\
1.05,1.09,1.2\end{array}$ & $\begin{array}{c}0.5 \text { (plain), } \\
\left.0.5 \text { (rotation } 10^{\circ}\right), \\
0.5(\text { scaling } 1.09)\end{array}$ \\
\hline The number of image & 48 & $48 \times 8=384$ & $48 \times 8=384$ & $48 \times 3=144$ \\
\hline
\end{tabular}

Table 2. Detection results of the plain copy-move forgery at the pixel level

\begin{tabular}{|c|c|c|c|}
\hline Algorithms & Precision $(\%)$ & Recall $(\%)$ & $F_{1}(\%)$ \\
\hline Emam [7] & 88.5 & 76.9 & 82.3 \\
\hline Shivakumar [16] & 68.1 & 76.4 & 72.0 \\
\hline Zhong [22] & 90.4 & 82.7 & 86.4 \\
\hline Pun [14] & 97.2 & 83.4 & 89.9 \\
\hline Proposed algorithm & 94.1 & 85.1 & 89.4 \\
\hline
\end{tabular}



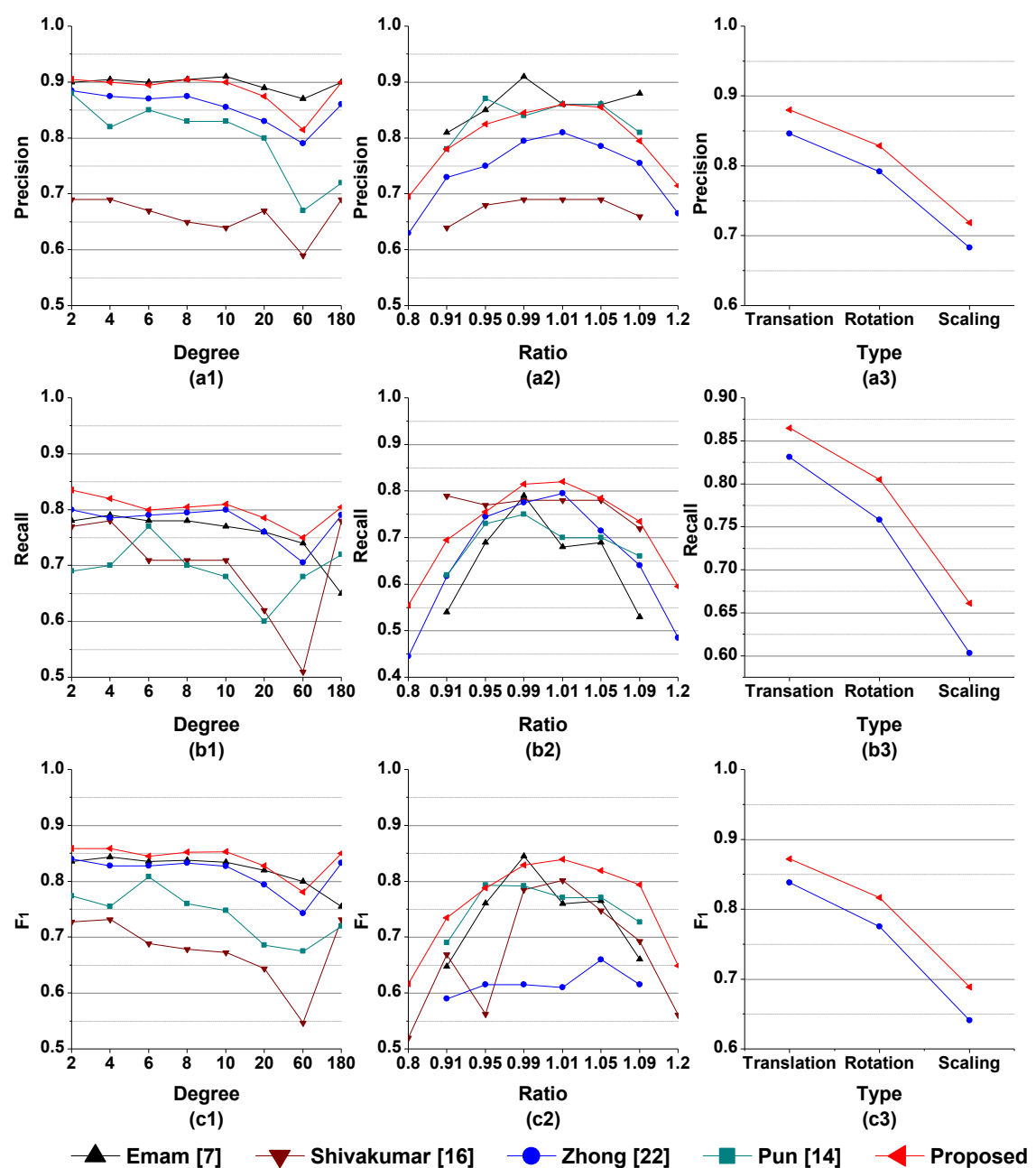

Figure 6. Performance comparisons between the proposed algorithm and the state-of-the-art algorithms on resisting rotation, scaling and down-sampling transforms: first to third rows depict the precision, recall and F1 scores, respectively; First to third columns depict the rotation, scaling and down-sampling transforms, respectively.

It is observed from Table 2 that the proposed algorithm achieved the best recall score of $85.1 \%$. The precision and $F_{1}$ score of the proposed algorithm is only a little lower than that of the algorithm of Pun [14]. It means that the proposed algorithm has superior performances compared to those of state-of-the-art algorithms.

Figure 6 shows the performances comparisons between the proposed algorithm and the state-of-the-art algorithms on resisting rotation, scaling and down-sampling transforms. As shown in Figure 6(a1), the anti-rotation performance of the proposed algorithm is a little lower than that of algorithm [7] at precision. However, the recall of the proposed algorithm, as shown in Figure 6(b1), got the best performance. The $F_{1}$ score also achieved the best performance in most cases. The antiscaling performance of the proposed algorithm achieved similar cases to anti-rotation performance as shown in Figure 6(a2)(c2). The $F_{1}$ score of the proposed algorithm as shown in Figure 6(c2) also achieved the best performance in most cases, except the scaling factor $=0.99$. Figure $6(\mathrm{a} 3)$, (b3) and (c3) show the precision, recall and $F_{1}$ score of down-sampling performances at translation, rotation $10^{\circ}$ and scaling factor 1.09. The performance of the proposed algorithm is better than that of the block-based algorithm [22] that employed the RHFMs to extract the image feature. According to the abovementioned analytical results, the proposed algorithm has superior performance than that of the state-of-the-art algorithms in resisting plain, rotation, scaling transforms and down-sampling etc.

\section{Conclusions}

In the view of the copy move forgery detection, many state-of-the-art algorithms have been proposed to deal with it. Unfortunately, most of the existed algorithms only do well in some simple geometric transforms, such as translation transform etc. Most of them are incompetent to resist other various geometric transforms. In this paper, SIFT is employed to extract features from pre-processing image. The 2NN test with Euclidean distance and RANSAC remove most outlier matches 
adaptively. Subsequently, the block features of the candidate matches are calculated by using RHFMs. The block features with their affine matrixes are evaluated to determinate criterion of the authentic inlier matches. The inliers matches are obtained. Then, SLIC is presented to segment the host image into texture blocks. The circular block is slid in the corresponding texture blocks that the texture blocks contain the inlier matches to further evaluate pixels for getting more corresponding matches. Finally, some geometric image operations, such as dilation, are employed to eliminate the small holes or isolated pixels. The detected regions are indicated by the white pixels. The experimental results show that the proposed algorithm can achieve the better performance than the existed state-of-the-art algorithms.

\section{Acknowledgements}

This work is supported by the 2017 Guangdong province university students scientific and technological innovation cultivation fund - Copy-move forgery image detection based on block-based algorithm (pdjh2017a0575).

\section{References}

1. R. Achanta, A. Shaji, K. Smith, A. Lucchi, P. Fua, and S. Süsstrunk, "SLIC Superpixels Compared to State-of-The-Art Superpixel Methods," IEEE transactions on pattern analysis and machine intelligence, vol. 34, no. 11, pp. 2274-2282, 2012.

2. I. Amerini, L. Ballan, R. Caldelli, A. D. Bimbo, and G. Serra, "A SIFT-Based Forensic Method for Copy-Move Attack Detection and Transformation Recovery," IEEE Transactions on Information Forensics and Security, vol. 6, no.3, pp. 1099-1110, 2011.

3. I. Amerini, L. Ballan, R. Caldelli, A. Del Bimbo, L. Del Tongo, and G. Serra, "Copy-Move Forgery Detection and Localization By Means Of Robust Clustering With J-Linkage," Signal Processing: Image Communication, vol. 28, no. 6, pp. 659-669, 2013.

4. E. Ardizzone, A. Bruno, and G. Mazzola, "Copy-Move Forgery Detection by Matching Triangles of Keypoints," IEEE Transactions on Information Forensics and Security, vol. 10, no. 10, pp. 2084-2094, 2015.

5. V. Christlein, C. Riess, and E. Angelopoulou, "A Study On Features for The Detection Of Copy-Move Forgeries," in Sicherheit, vol. 2010, pp. 105-116, 2010.

6. V. Christlein, C. Riess, J. Jordan, C. Riess, and E. Angelopoulou, "An Evaluation of Popular Copy-Move Forgery Detection Approaches," IEEE Transactions on Information Forensics and Security, vol. 7, no. 6, pp. 1841-1854, 2012.

7. M. Emam, Q. Han, and X. M. Niu, "PCET Based Copy-Move Forgery Detection in Images Under Geometric Transforms," Multimedia Tools and Applications, vol. 75, no. 18, pp. 11513-11527, 2016.

8. A. J. Fridrich, B. D. Soukal, and A. J. Lukáš, "Detection of Copy-Move Forgery In Digital Images," in Proceedings of Digital Forensic Research Workshop, 2003.

9. H. Huang, W. Guo, and Y. Zhang, "Detection of Copy-Move Forgery in Digital Images Using SIFT Algorithm," in Proceedings of 2008 IEEE Pacific-Asia Workshop on Computational Intelligence and Industrial Application, vol. 2, pp. 272-276, 2008.

10. T. Huynh-Kha, T. Le-Tien, S. Ha-Viet-Uyen, and K. Huynh-Van, "The Efficiency of Applying DWT And Feature Extraction Into Copy-Move Images Detection," in Proceedings of 2015 International Conference on Advanced Technologies for Communications (ATC), pp. 44-49, 2015.

11. X. Kang and S. Wei, "Identifying Tampered Regions Using Singular Value Decomposition in Digital Image Forensics," in Proceedings of 2008 International Conference on Computer Science and Software Engineering, vol. 3, pp. 926-930, 2008.

12. W. Luo, J. Huang, and G. Qiu, "Robust Detection of Region-Duplication Forgery In Digital Image," in Proceedings of 18th International Conference on Pattern Recognition, vol. 4, pp. 746-749, 2006.

13. A. C. Popescu and H. Farid, "Exposing Digital Forgeries by Detecting Traces Of Resampling," IEEE Transactions on signal processing, vol. 53, no. 2, pp. 758-767, 2005.

14. C. M. Pun, X. C. Yuan, and X. L. Bi, "Image Forgery Detection Using Adaptive Oversegmentation and Feature Point Matching," IEEE Transactions on Information Forensics and Security, vol. 10, no. 8, pp. 1705-1716, 2015.

15. J. Qin, F. Li, L. Xiang, and C. Yin, "Detection of Image Region Copy-Move Forgery Using Radial Harmonic Fourier Moments," Journal of Image \& Graphics, vol. 18, pp. 919-923, 2013.

16. B. Shivakumar and L. D. S. S. Baboo, "Detection of Region Duplication Forgery In Digital Images Using SURF," International Journal of Computer Science Issues, vol. 8, no. 4, pp. 199-205, 2011.

17. P. WANG, N. CAO, X.-c. CAO, and J.-f. OUYANG, "Fast Random Sample Consensus Based on Cross Ratio Invariance," Journal of Tianjin University, vol. 2, p. 017, 2011.

18. Y. Wo, K. Yang, G. Han, H. Chen, and W. Wu, "Copy-Move Forgery Detection Based On Multi-Radius PCET," IET Image Processing, vol. 11, no.2, pp. 99-108, 2016.

19. M. Zandi, A. Mahmoudi-Aznaveh, and A. Talebpour, "Iterative Copy-Move Forgery Detection Based on a New Interest Point Detector," IEEE Transactions on Information Forensics and Security, vol. 11, no. 11, pp. 2499-2512, Nov 2016.

20. F. Zhao, R. Zhang, H. Guo, and Y. Zhang, "Effective Digital Image Copy-Move Location Algorithm Robust To Geometric Transformations," in Proceedings of 2015 IEEE International Conference on Signal Processing, Communications and Computing, pp. 1-5, 2015.

21. J. Zheng, Y. Liu, J. Ren, T. Zhu, Y. Yan, and H. Yang, "Fusion of Block And Keypoints Based Approaches For Effective CopyMove Image Forgery Detection," Multidimensional Systems and Signal Processing, vol. 27, no. 4, pp. 989-1005, 2016.

22. J. Zhong, Y. Gan, and S. Xie, "Radon Odd Radial Harmonic Fourier Moments In Detecting Cloned Forgery Image," Chaos, Solitons \& Fractals, vol. 89, pp. 115-129, 2016. 
23. J. Zhong, Y. Gan, J. Young, and P. Lin, "Copy Move Forgery Image Detection via Discrete Radon and Polar Complex Exponential Transform-Based Moment Invariant Features," International Journal of Pattern Recognition and Artificial Intelligence, vol. 31, no. 2, p. $1754005,2017$.

Yanfen Gan received her M.S. degree in signal and information processing from Guangdong University of Technology in 2007, China. She is currently a university associate professor in the school of information science and technology, at Guangdong University of Foreign Studies South China Business College, China. Her current research interests include information system security, pattern recognition, signal and image processing.

Jimlee Chung received his M.S. degree in Detection technology and automation devices from Sun Yat-sen University in 2006, China. He is currently a senior engineer in the school of information engineering at Guangdong Mechanical \& Electrical College, China. His current research interests include information system security, watermarking, pattern recognition, signal and image processing.

Janson Young is currently working toward the M.S. degree of software engineering from Guangdong University of Technology, China. His research interests are image processing and system modelling, algorithms and computer systems, pattern recognition.

Zixin Hu is a student in the school of information engineering at Guangdong Mechanical \& Electrical College, China. His research interests are image processing.

Jinhong Zhao received his M.S. degree in Mechanical and Electronic Engineering from Graduate University of Chinese Academy of Sciences in 2009, China. He is currently a lecturer in the school of information engineering at Guangdong Mechanical \& Electrical College, China. His current research interests include information system security, video and image processing. 